\title{
Research on the monitoring system for wire rope of window cleaning machine based on the technology of ZigBee
}

\author{
Yao Jinmei ${ }^{1, a}$, Zou Defang ${ }^{2, b}$, Zhou Peng $^{1, \mathrm{c}}$ and Sun Jian ${ }^{2, \mathrm{~d}^{*}}$ \\ ${ }^{1}$ Graduate school, Shenyang Jianzhu University, China \\ ${ }^{2}$ Mechanical Engineering School, Shenyang Jianzhu University, China \\ ayaojinmei06@126.com, bzdfyy@163.com, czhoupeng0718@163.com, dsun503529@126.com
}

Keywords: wire rope, ZigBee, monitoring, node

Abstract. Background on modern safety monitoring of building maintenance equipment, the technology of ZigBee is applied. Steel wire rope monitoring system of window cleaning machine is researched in this paper. Its key question is to achieve low speed, low cost and low-power consumption. Star topology of wireless sensor network is formed to detect the health status steel wire rope of window cleaning machine based on the window cleaning machine. The results show that star topology on the wireless network monitor is feasible monitoring system for wire rope. Safe reliability of this equipment could be improved effectively. The characteristics of simple method, low power consumption, low cost are very suitable for low bit rate transmission. It must has broad application prospect by its technical features.

\section{Introduction}

With the progress of science and technology, and modern building mechanical devices are toward a larger and more complicated, automatic direction. And the factor which has a bigger effect on system operation is increased[1,2]. This structure makes the complexity of the systems and equipment become more and more complex. Therefore, when the efficiency of operation is improved, the production costs should be reduced. However, the machinery and equipment failures, shutdown caused not only the enormous economic losses, but also endanger people's personal safety[3,4]. In order to ensure the normal operation of the machinery and equipment, improving reliability and safety rates, the effective measure should be adopted. Running of the construction machinery and equipment to carry out effective monitoring and control should be enhanced. And the abnormal circumstance of the equipment could be discovered in due course. This paper has solved the problem of safety and reliability of the equipment effectively. To improve operational efficiency, to save unnecessary economic loss, promoting sustainable economic development in China, it plays a very important role.

Window cleaning equipment belongs to high altitude flying machines. The connecting type between gondola and suspension model of this machine is wire rope connection[5]. If the steel wire rope there is a problem, directly affects the security of staff in the gondola, as well as the safety of ground personnel[6]. So, suspended wire rope health monitoring is particularly important. Detecting the steel wire rope of window cleaning equipment by wire forms is difficult. For one reason, wiring is a technical difficulty. For the other reason, cable increases the weight and cost of the gondola. Based on this, the author uses wireless monitor for real-time monitoring of the admissibility of wire rope. Research on understanding gondola wire monitoring system based on ZigBee technology is of great reference value. 


\section{The system and principle of monitoring of wire rope}

Online monitoring equipment is installed at the time of steel wire rope running. This monitoring and supervision system could instrumental operation and self-diagnostics at the condition of no interference and unsupervised. Online monitoring system is constructed by on-line monitoring system, data collection and disposal system and computer system[7-9]. On-line monitoring system of wire rope is shown as Fig. 1.

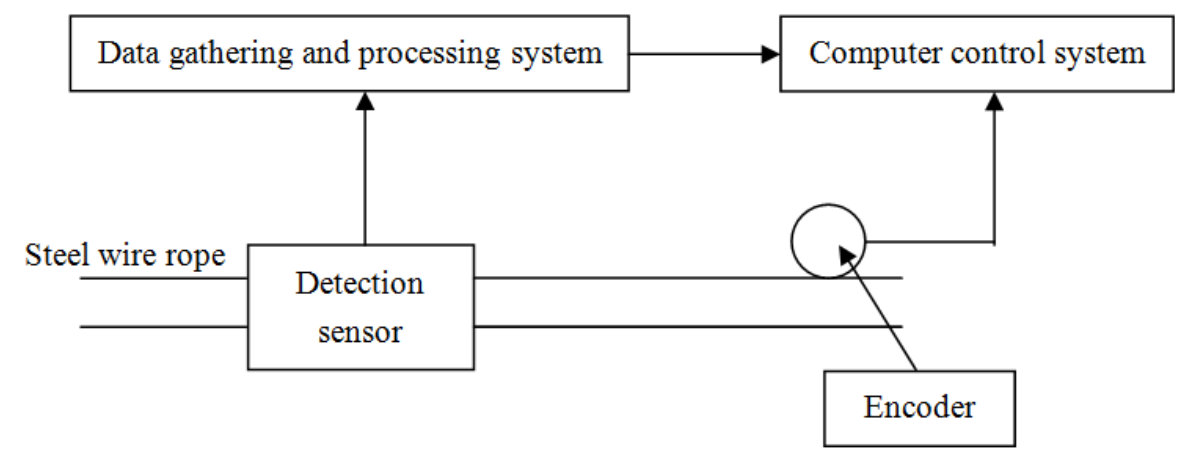

Figure 1 On-line automatic monitoring system of wire rope

Online monitoring system for steel wire rope is mainly used for on-line real time monitoring of wire rope damage. Monitoring signal is then passed to data acquisition and processing system by this monitoring system. The system detected signal transmission to the computer system and related analysis.

\section{The technology of ZigBee}

\section{Technical characteristics of ZigBee}

ZigBee technology is a low-speed, short-distance wireless local area network technology. This standard makes the low power consumption, low traffic rate, low cost, as an important goal. There are three frequencies of operation on Direct Sequence Spread Spectrum(DSSS) by ZigBee technology. They are $868 \mathrm{MHz}, 915 \mathrm{MHz}$ and $2.4 \mathrm{GHz}$ respectively and they are required to apply licensed frequency bands[10-12]. It has a short range, low complexity, low power consumption, low cost, low data rates, flexible installation and so on. ZigBee technology is ideal for communication amount of data is small, the data transfer efficiency is relatively low cost and power consumption is very low. So, features of ZigBee technology will decide it is the best choice for wireless network technology. It is widely used in the field of construction sites, industrial control equipment, industrial wireless location control and so on[13-14]. The advantage of the ZigBee is shown as:

(1) Reliability. The MAC layer of ZigBee uses the collision avoidance mechanism. When there are data sent, the recipient receives the information back and confirms the information. If the receiver do not receive acknowledgement from the receiver, this means that packets sent during the collision. So, another sending is required. In this way to ensure the reliability of the system.

(2) Short delay. Sensitive applications of the time delay are optimized pertinently. The communication delay and hibernation is activated for a short period of time delay.

(3) Power saving. Operation life of this equipment could be sustained 6 months to 2 years by two fifth batteries.

(4) Safety. The Protocol of ZigBee provides data integrity and authentication functionality, it provides three levels of security in data transmission.

(5) Network capacity. Nodes of the network at the same time work up to 65000. 


\section{Network topologies of ZigBee}

There are three types for the topological structure of the networks in ZigBee wireless network. They are star topology, tree-type networks structure and MESH-type network structure shown as Fig. 2. Depending on how the structure and application of window cleaning machine, the logical location of sensor nodes is invariant. The process of networking is simple, and the data traffic is not very big. So, coordinator and terminal nodes of a star network topology will be able to complete the proper communication of the data.
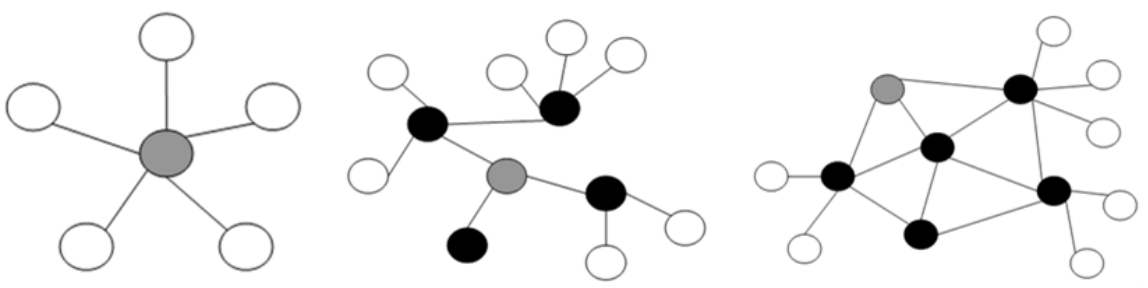

(a) Star topology (b) Tree-type networks structure (c) MESH-type network structure Figure 2 Network topologies of ZigBee

Depending on how the structure and application of window cleaning machine, this paper used a star network topology to gondola wire rope online real-time monitoring.

\section{The architecture of ZigBee agreement}

Protocol suite of ZigBee is established on the standard of PHY layer and MAC sub layer of IEEE 802.15.4. The communicating between network layer and application layer is realized by it. The application support sub layer and ZigBee device object are provided by application layer. The application framework has been incorporated in the user-defined application objects.

The architecture of ZigBee is constituted by the layer from every module. Each layer provides specific services for the upper. It is meaning that entities from the data service providing data transmission services. Management entity is applied with all the other management services. Each service entity through the appropriate service access point (SAP) to its upper layer provides an interface, each access point corresponding to the complete functionality by service primitives. The architecture of ZigBee agreement is shown as Fig. 3.

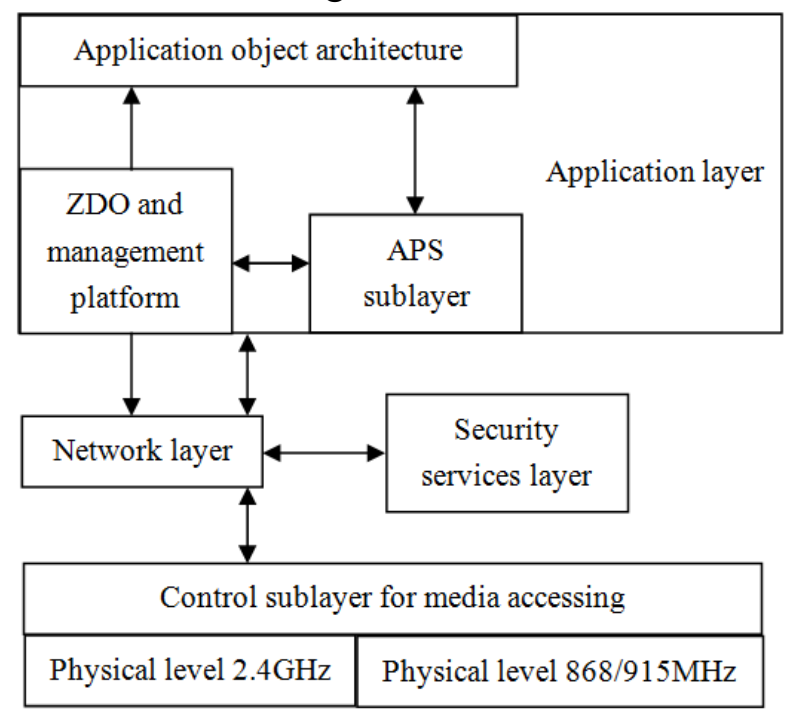

Figure 3 Architectural model of ZigBee

\section{System on chip of CC2530}

RF Chips CC2530 is used to set up wireless communication module in this design. CC2530 is a System on Chip for 2.4GHz IEEE5802.15.4、ZigBee and ZigBee RF4CE. The chip of CC2530 needs to buy a few external components to work stable. It has three types of models. They are 
memory module, peripheral modules and power-supply module. Due to wireless communication module of impressive features and very few external components, it can be such advantages as steady work. The chip is widely used in home control, building automation, industrial control, lighting, consumer electronics, low power wireless sensors, medical fields and so on. Its structure diagram is shown as Fig. 4.

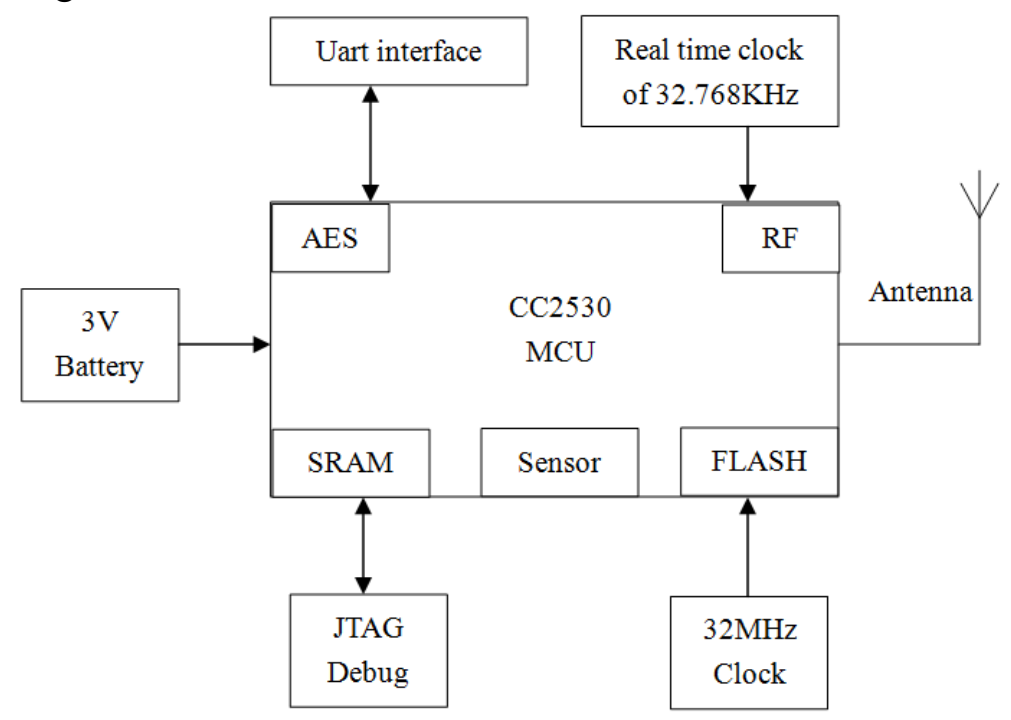

Figure 4 Hardware structure diagram of the node

In practical application, due to the long run requiring hardware is simple so that hardware can reduce power consumption. The whole hardware structure of system is very simple. And it just includes the following several parts: system on chip of CC2530, real time clock of $32.768 \mathrm{KHz}$, system clock of $32 \mathrm{MHz}$, serial port, java debug, antenna and power supply. So look on the whole, CC2530 system is the main energy consumers, and the other value of energy consumption could be ignored. Also from the previous chapter in the CC2530 introduction to system on chip, CC2530 typical consumption power consumption is very low, therefore, the system is power. It can meet the requirements node runs for a long time.

\section{Summary}

With the rapid development of construction equipment industry, higher requirements of monitoring technology for construction machinery are raised by the user. And great changes have taken place in its monitoring methods. Advanced control theory and technology are used to eliminate the problems and flaws when monitoring system is working. So, the security and stability of construction machinery are improved. The technical features of ZigBee are analyzed in this paper. The application of wire rope detect system of window cleaning machine is researched based on the technology of ZigBee. Through this study found a real-time understanding of the health condition of wire rope is now the key problems to be solved. With the development of Wireless Networks, on-line monitoring of steel rope system is very important. In a word, wire rope on-line automatic monitoring system remains to be further investigated, especially the problem in monitoring of window cleaning machine. High accuracy of on-line monitoring and alarm system of wire rope needs to be developed.

\section{References}

[1] Hou Fengtao. Kinematics and Integration Finite Element Analysis of Goose-neck Jib Gondola CD250[D]. Xi'an: Chang'an University, 2009. 
[2] LAN Yangchun, XUE Baoxin. Design of building maintenance units[J]. CONSTRUCTION MECHANIZATION, 2010(7): 43-46.

[3] WU An, CAO En-qin, LIU Yun-hui. Exploitation of a sort of goose-neck jib window cleaning equipment for highrise building[J]. CONSTRUCTION MECHANIZATION, 2007(2):26-28.

[4] Zhang Hua, Dong Wei, Wu Hai. Protection mechanism of reserve for window cleaning equipment[J]. CONSTRUCTION MECHANIZATION, 2009(8):35-38.

[5] XI Hui, MA Jun-xing, WANG Jin, FANG Xiao-jun, LI Peng, LIU Yu-jian, XIE Dan-lei. Finite element analysis of base and column of goose-neck jib gondola[J]. CONSTRUCTION MECHANIZATION, 2010(7):50-52.

[6] Li Wenzhong, Duan Chaoyu. Entrance and practice for Wireless Network Technology of ZigBee[M]. Beijing: Beijing University of Aeronautics and Astronautics Press, 2007.

[7] ANG Zhimin,JIN Haihong,FAN Zhiguo,DUAN Yong. WSN Node Design and Communication Realization Based on ZigBee Protocol[J]. MODERN ELECTRONICS TECHNIQUE. 2007,249:47-50.

[8] Ren F. Wireless sensor networks[J]. Journal of Software, 2003,14(7): 1282-291.

[9] Kadu S D,Deshpande V S. Handeling Throughput in wireless sensor network[J].Computational Intelligence \& Computing Research (ICCIC),2012 IEEE International Conference on, 2012, 8350(2): $1-4$.

[10]Ju X,Zhang H, Sakamuri D. NetEye: a user-centered wireless sensor network testbed for high-fidelity robust experimentation [J].International Journal of Communication Systems, 2012, 25(9): 1213-1229.

[11]Jiang P. A New Method for Node Fault Detection in Wireless Sensor Networks[J]. Sensors (Basel)., 2009, 9(2): 1282-1294.

[12]Fu Yunqing, Wang Songjian, Wu Zhongfu. Routing protocol of wireless mesh network based on weighted link state[J]. Computer Research and Development, 2009, 1(46): 137-143.

[13] S. Ni, Y. Tsing, Y. Chen, et al. The broadcast storm problem in a mobile ad hoc network, Wireless Networks, 2002, 8(2-3): 153-167.

[14] Kim Yu-Doo, Moon II-Young. Improved AODV routing protocol for wireless sensor network based on ZigBee. In: 11th International Conference on Advanced Communication Technology, Phoenix Park, Korea, 2009, 859-862. 